\title{
Abacus System for Ghanaian basic Schools
}

\author{
S. Alhassan \\ Regentropfen College of Applied \\ Science \\ Department of Computer Science \\ Bolgatanga, Ghana
}

\author{
M. O. Yinyeh \\ University for Development \\ Studies \\ Faculty of Mathematical Sciences \\ Department of Computer Science
}

\author{
G. K. Armah \\ University for Development \\ Studies \\ Faculty of Mathematical Sciences \\ Department of Computer Science
}

\begin{abstract}
The abacus is an instrument that makes solving of problems of addition, subtraction, multiplication and division very fast and efficiently with less effort. Despite its numerous benefits, most Ghanaian Basic schools teachers resort to the use of "counters", "pebbles" or "sticks" system to teach basic mathematics of addition, subtraction, multiplication and division. In this paper, we designed an Abacus system of seven (7) rods to help Ghanaian Basic school pupils learn to solve arithmetic problems between zero (0) and one million $(1,000,000)$. The system contains well-structured lessons and user-friendly interfaces designed to encourage the student to learn to solve arithmetic operations.
\end{abstract}

\section{General Terms}

Abacus, Suanpan, Soroban, beam, rod, clear, ones rod, tens rod, hundreds rod.

\section{Keywords}

Abacus, Chinese Suanpan, Japanese Soroban, heaven beads, earth beads

\section{INTRODUCTION}

The Abacus (or Soroban in Japan or Suanpan in Chinese) is an ancient mathematical instrument used for calculation. It is one of the world's first real calculating tools - and early forms of an Abacus are nearly 2500 years old. Abacus is a Latin word meaning sand tray. The word originates with the Arabic "abq", which means dust or fine sand. In Greek it is called "Abax" or "abakon" meaning table or tablet. The original types of Abacus were stone slates with dust covering them and a stylus used for marking numbers. Later this evolved into a slate with groves where rocks or other counters would be placed to mark numbers. Later it finally evolved into a framed device with beads sliding along bamboo rods. The frame is divided into an upper and lower section by a bar called the "beam" [1-10]. The abacus can be used for addition, subtraction, multiplication, and division.

\section{KINDS OF ABACUSES}

The abacus is divided into two sections; the upper part called "heaven beads" has a value of 5 each and the lower part called "earth beads" has a value of 1 each [1-10]. In this work, we discussed the Chinese Suanpan and the Japanese Soroban. The Japanese "Soroban" has one bead on the upper section of each rod and five/four beads on the lower section of each rod as shown in Figure 1 and Figure 2.

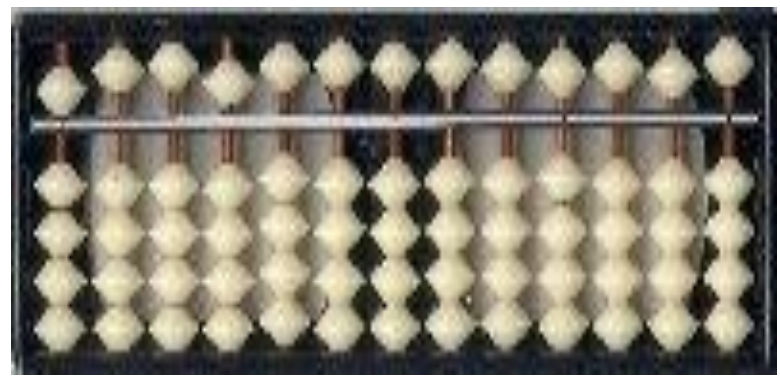

Figure 1: Japanese Soroban with $(4,1)$ beads on each rod

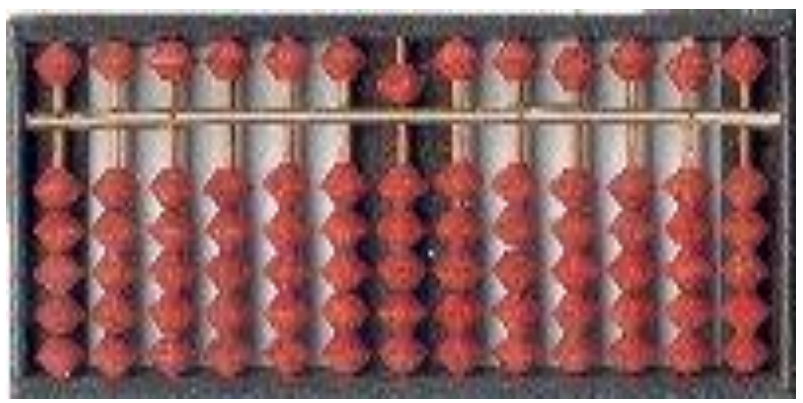

Figure 2: Japanese Soroban with $(5,1)$ beads on each rod

On the Chinese "Suanpan", there are two beads on the upper section of each rod and five beads on the lower section of each rod. This is depicted in Figure 3.

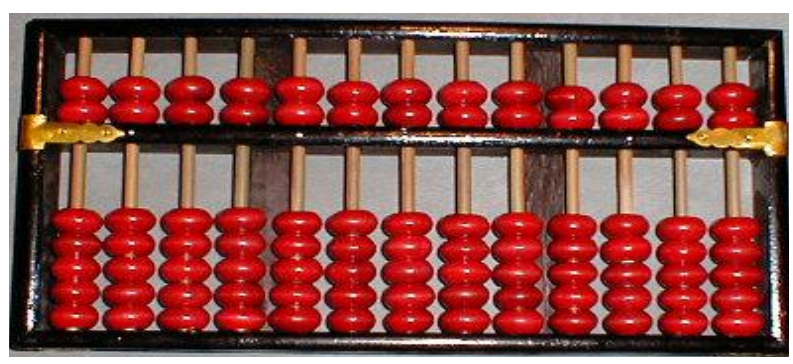

Figure 3: Chinese Suanpan with $(5,2)$ beads on each rod

\section{USING ABACUS}

\subsection{Setting Numbers on the Abacus}

Before using the abacus it must be cleared. This is done by moving all of the "heaven beads" to the top of the frame and all of the "earth beads" to the bottom of the frame. 


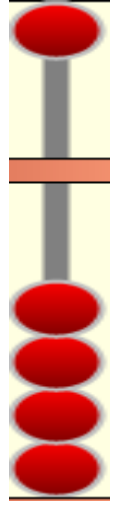

Figure 4: Earth and heaven beads cleared

A bead is said to "have value" (set) when it is pushed towards the middle of the rod and "loses value" when moved away from the middle rod.

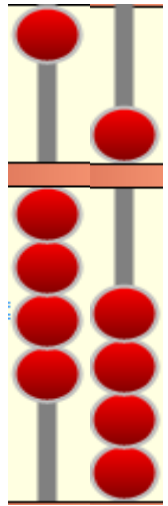

Figure 5: Earth beads (left) and Heaven bead (right) set

The abacus is read by counting the beads that have been set. In Figure 6 below, the value on the rods starting from the left are $1,3,8$ and 11,268 .

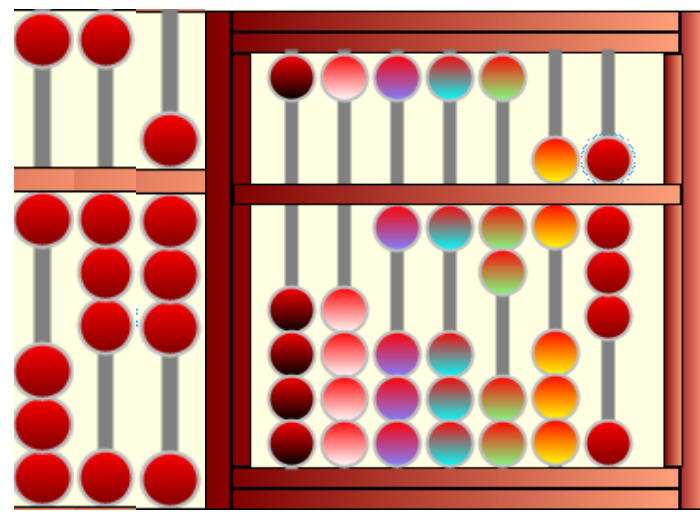

Figure 6: Reading abacus

\subsection{Addition in Abacus}

Problems of addition can simply be solved with abacus by setting beads and the use of complementary numbers with respect to 5 and 10. Addition in abacus is done from the lowers rod to the highest rod (i.e. right to left).

For example; add $8+4$

\section{Solution}

Step 1: Set 4 on 1s rod

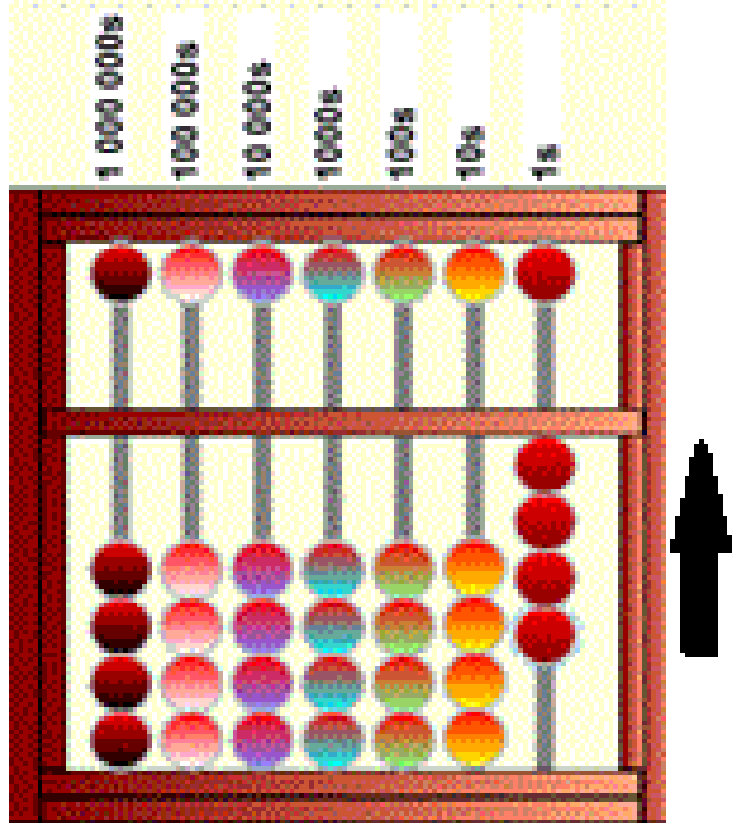

Figure 7: 4 set on 1s rod

Step 2: Add 8.

Because the 1s rod does not have 8 available, we use the complementary number for 8 with respect to 10 . That is 2 . Hence, subtract the complementary 2 from 4 on the 1 s rod and carry 1 to the $10 \mathrm{~s}$ rod. This gives the final results of 12 . Thus $4+8=12$

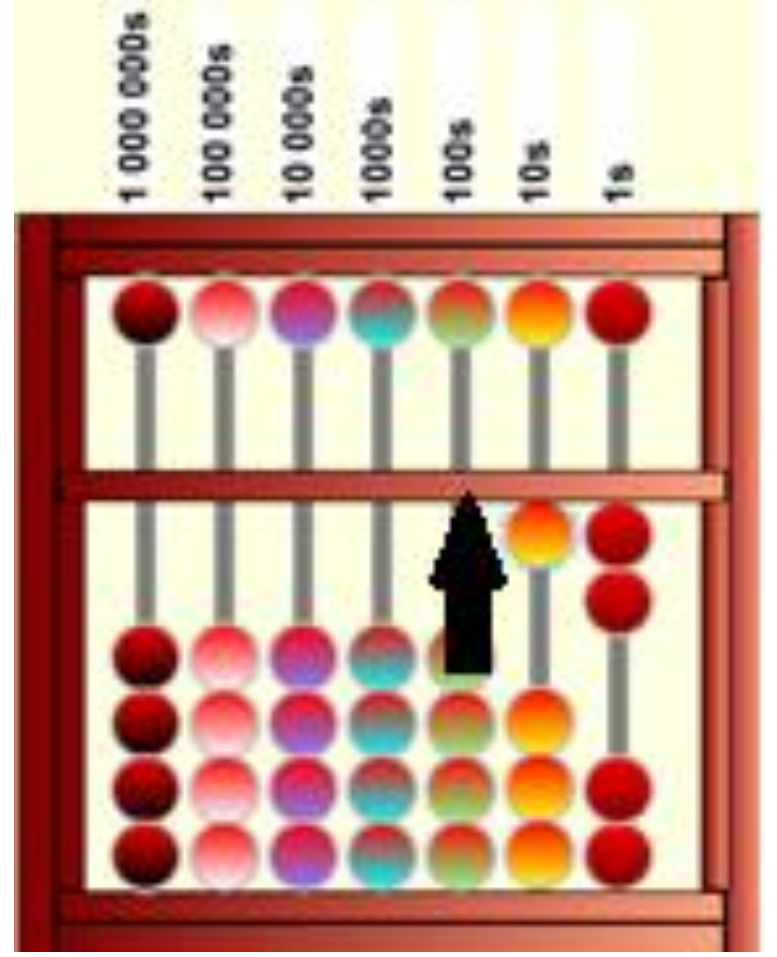

Figure 8: Complementary 2 subtracted from 1 s rod and Carry 1 to 10s rod

\subsection{Subtraction in Abacus}

Solutions to subtraction problems using abacus is achieved by always adding the complement. The order of subtraction is from the rods with the highest units to the lowers unit (i.e. left to right). 
For example; subtract 13-7

\section{Solution}

Step 1: Set 13 on 1 s and 10 s rods

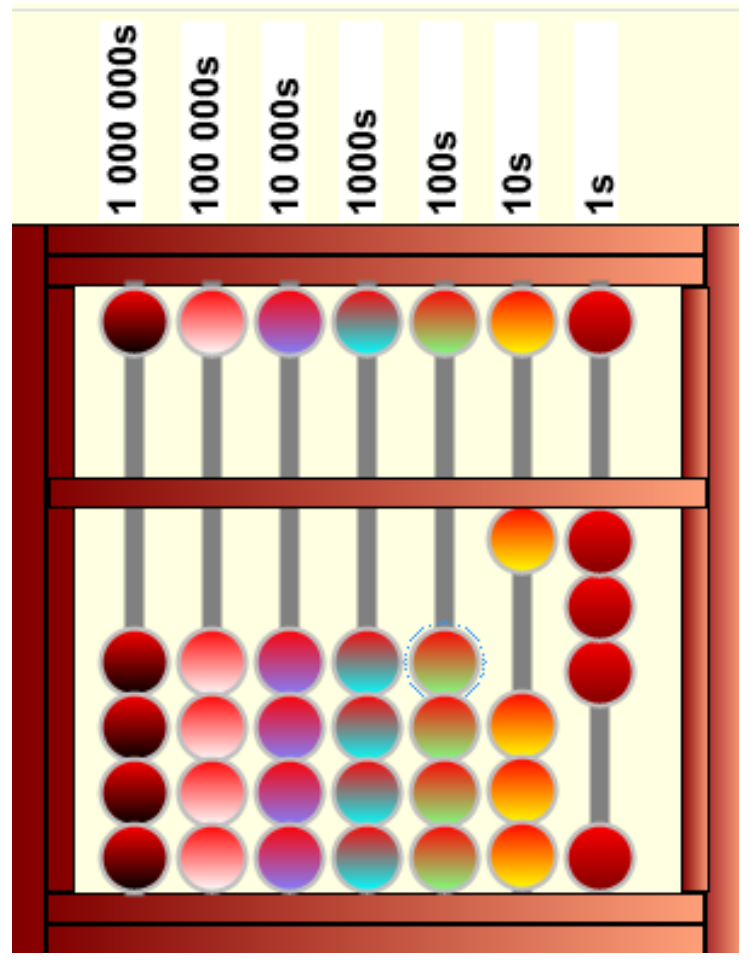

Figure 9: 13 set on 1s and 10s rods

Step 2: Subtract 7.

Since rod 1 only carries the value 3 , we use the complementary number of 7 with respect to 10 , which is 3 . Hence, we subtract 1 from the 10 s rods and add 3 to the $1 \mathrm{~s}$ rod (move 2 earth beads away from the beam and move 1 heaven bead towards the beam) to make 6 . Therefore, 13-7=6.

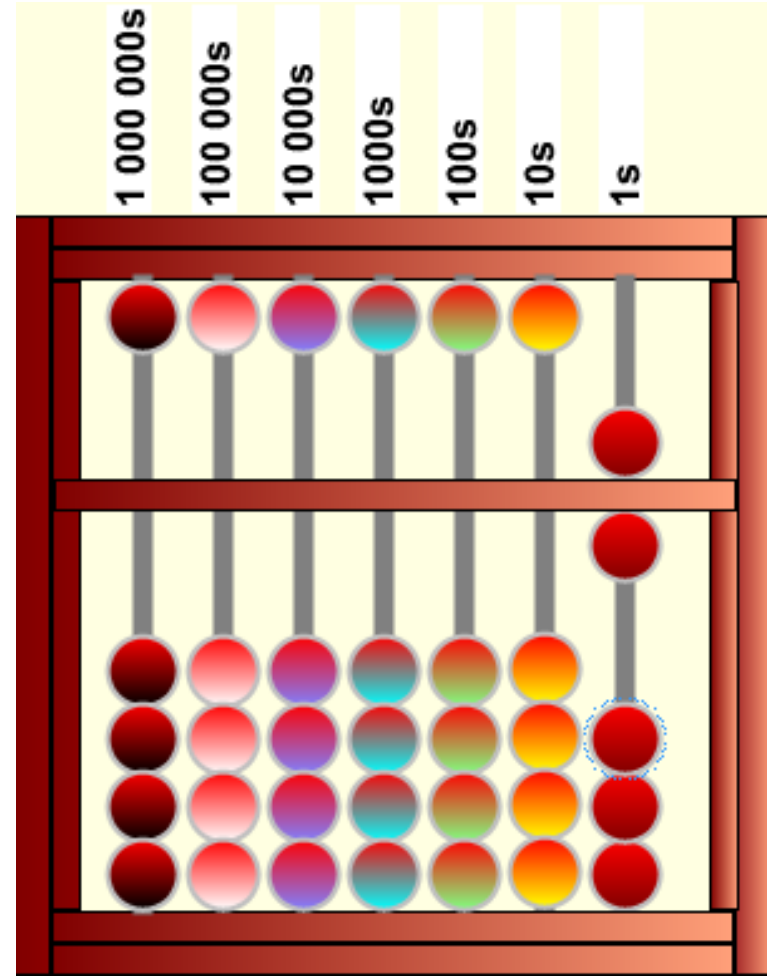

Figure 10: Subtract 1 from 10s rod and add 3 to $1 \mathrm{~s}$ rod to get 6

\subsection{Multiplication in Abacus}

Before multiplication problems can be solved with abacus, pre-defined positions of the multiplicand and the multiplier is determined. In this work, we set the multiplicand on the Millions, Hundred Thousands and Ten Thousands rods and use the Thousands rod to set the multiplier. The answer is read from the Tens and Ones rods.

For example; multiply 14 × 6

\section{Solution:}

Step 1: Set 14 on Millions and Hundred Thousands Rods. Step 2: Set 6 on Thousands Rod.

Figure 11 shows Steps 1 and 2.

Step 3: Multiply 6 by 1 to get 6 and Set on Hundreds and Tens Rods.

Step 4: Multiply 6 by 4 to get 24 and Set on Tens and Ones Rods.

Figure 12 shows Steps 3 and 4. 


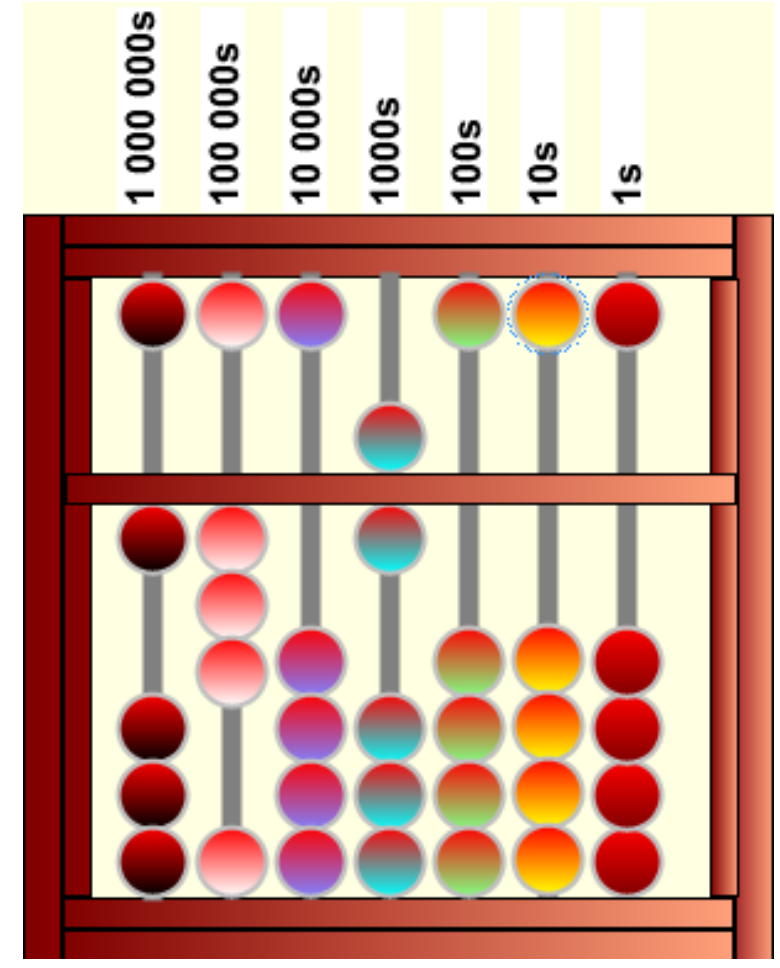

Figure 11: Representation of steps 1 and 2.

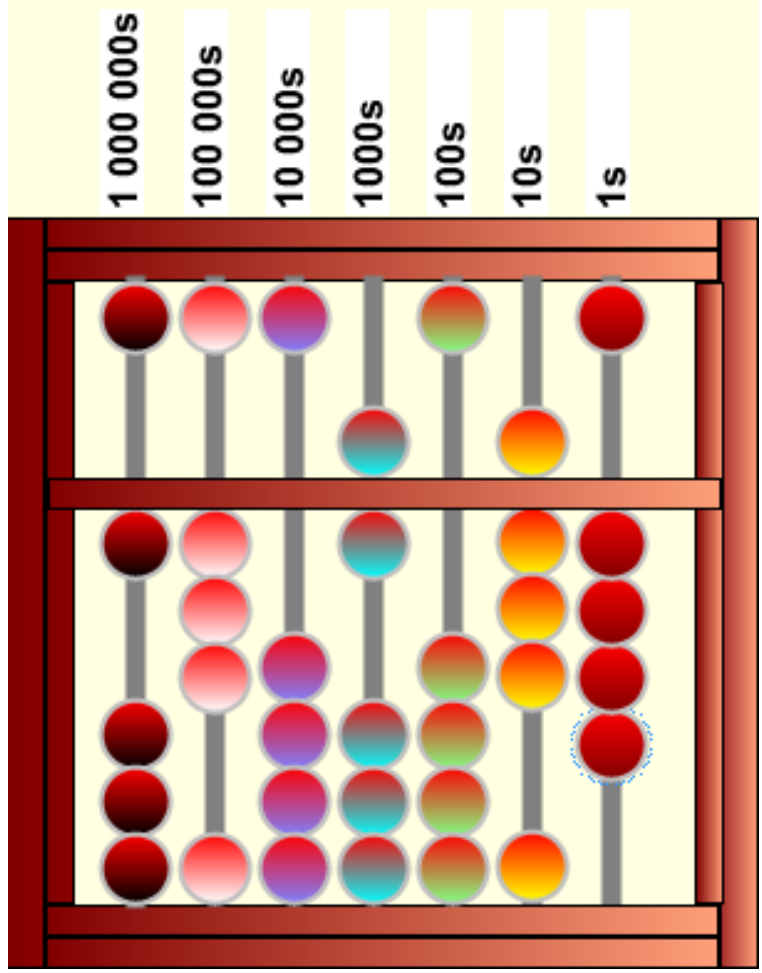

Figure 12: Representation steps 3 and 4.

Step 5: Clear the Millions, Hundred Thousands and Thousands Rods.

The answer is 84 . This is show in Figure 13

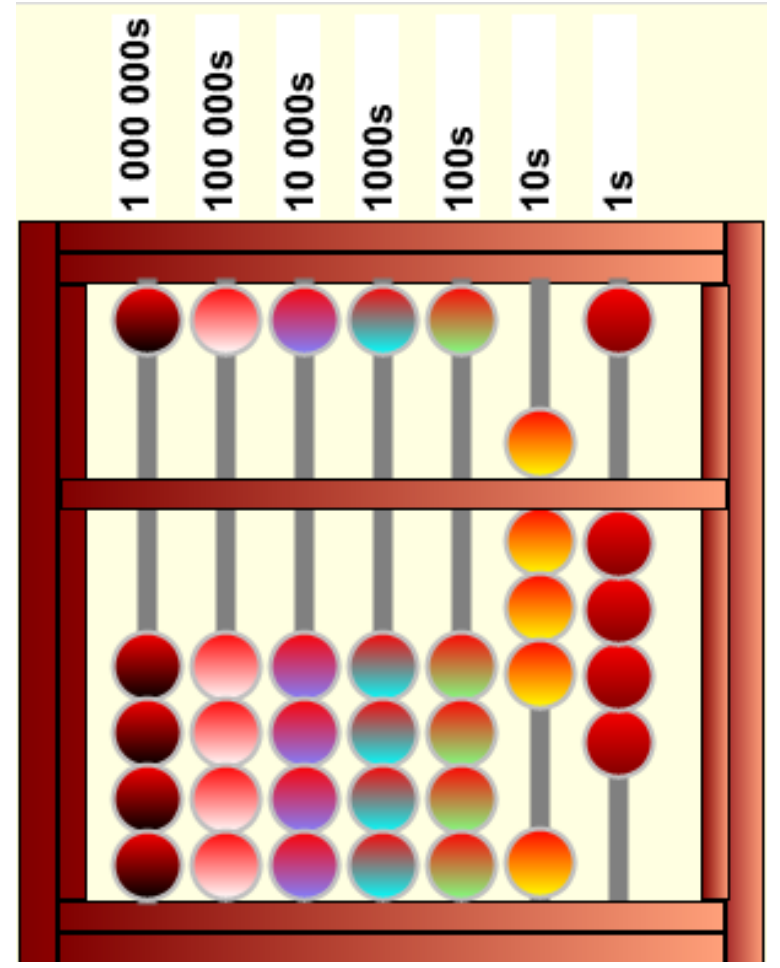

Figure 13: Final results of $14 \times 16=84$

\subsection{Division in Abacus}

With division, the dividend is set on the Hundreds, Tens and Ones rods whiles the divisor is set on the Millions and Hundred Thousands rods. The results of the division operations are set on the Ten Thousands and Thousands rods; and the answer read from left to right.

For example; divide $57 \div 3$

Step 1: Set 57 and 3 on Hundreds, Tens, Ones and Millions Rods. This is shown in Figure 14

Step 2: Set 1 on Ten thousands Rod. Thus, 5 divided by 3 gives 1 remainder 2 . The number reduces to 27 .

Step 3: Set 9 on Thousands Rod. Thus, 27 divided by 3 gives 9 remainder 0 . The number reduces to 0 . The representation of Steps 2 and 3 are shown in Figure 15. 


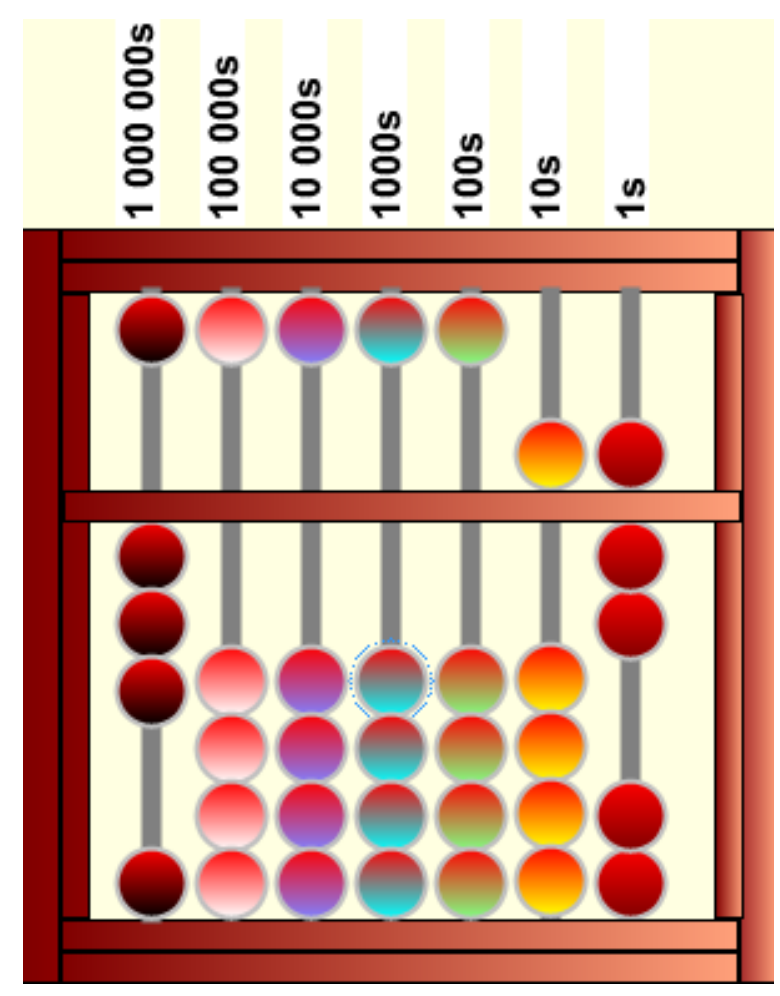

Figure 14: Presentation of step 1

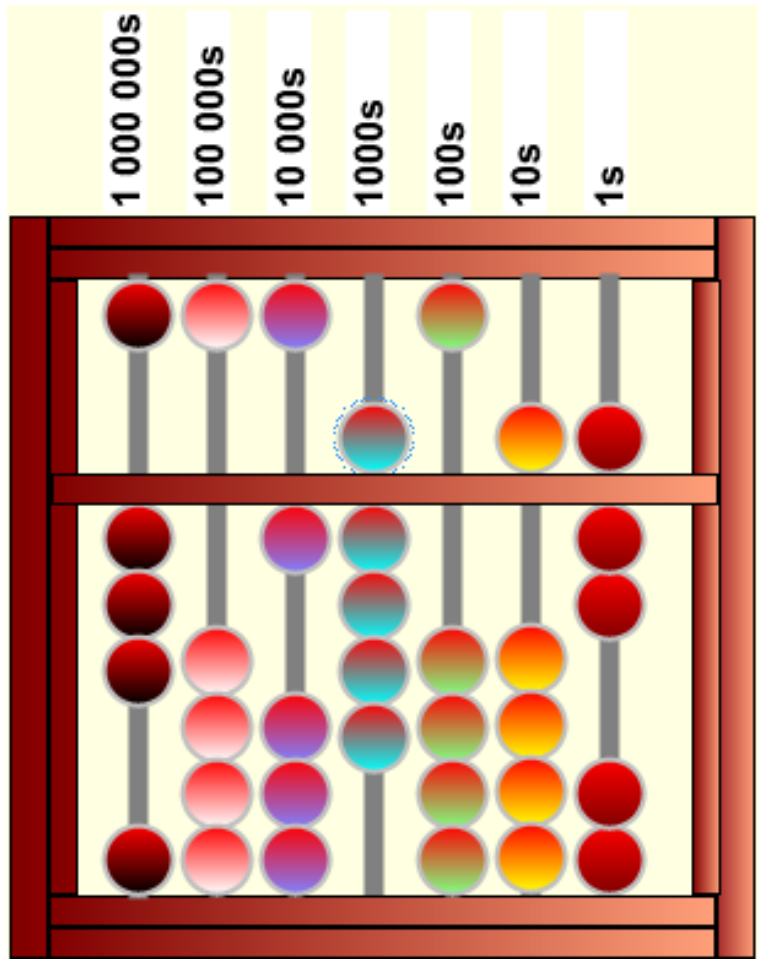

Figure 15: Presentation of steps 2 and 3

Step 4: Clear the Hundreds, Tens, Ones and Millions Rods.

The answer is 19. The final result is show in Figure 16.

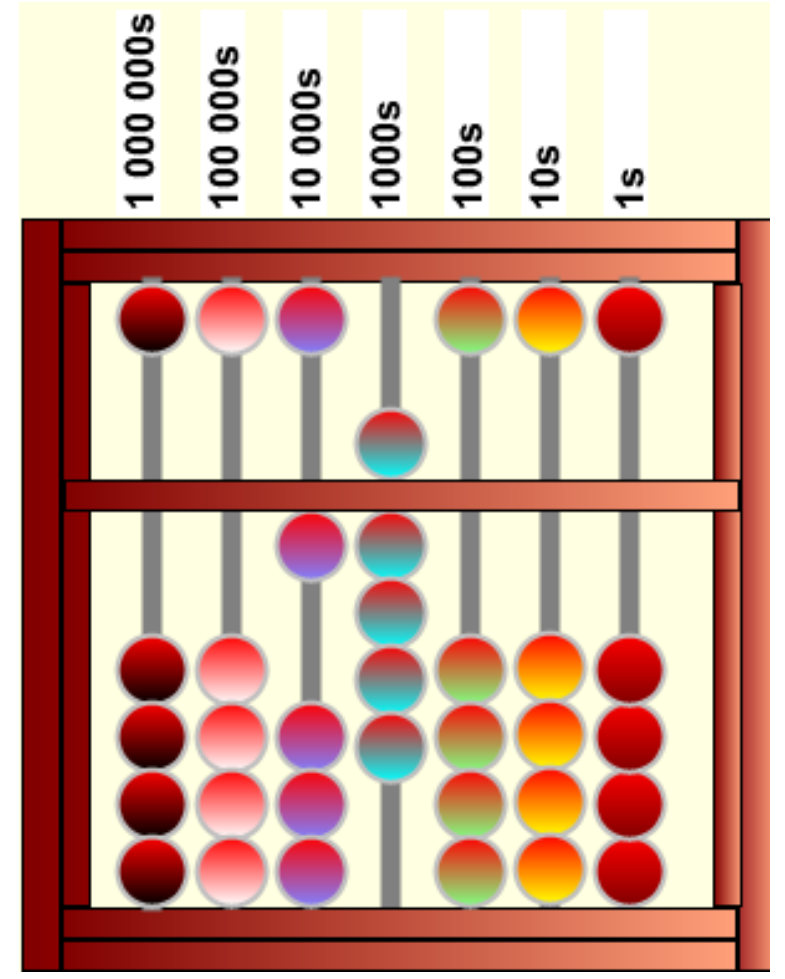

Figure 16: Final results of $57 \div 3=19$

\section{SYSTEM DESIGN AND IMPLEMENTATION}

The proposed software is developed in Visual Basic.Net with the incorporation of windows narrator that provides audio interactive facility to complement textual display of instructions. Adequate lessons, free practice and quizzes have been provided to handle both Soroban and Suanpan separately. Also, a help facility known as "show me" is included in the free practice activities to help the user solve problems after four (4) unsuccessful try of a problem.

\subsection{Sign in Window}

To make the software child-friendly, the Sign/Log in window only requires the user's name; no password is required. User registration allows the user to choose a user name, the type of abacus (Japanese Soroban or Chinese Suanpan) and the level (Beginner, Intermediate or Advance). Figure 17 shows the user selection/registration window. 


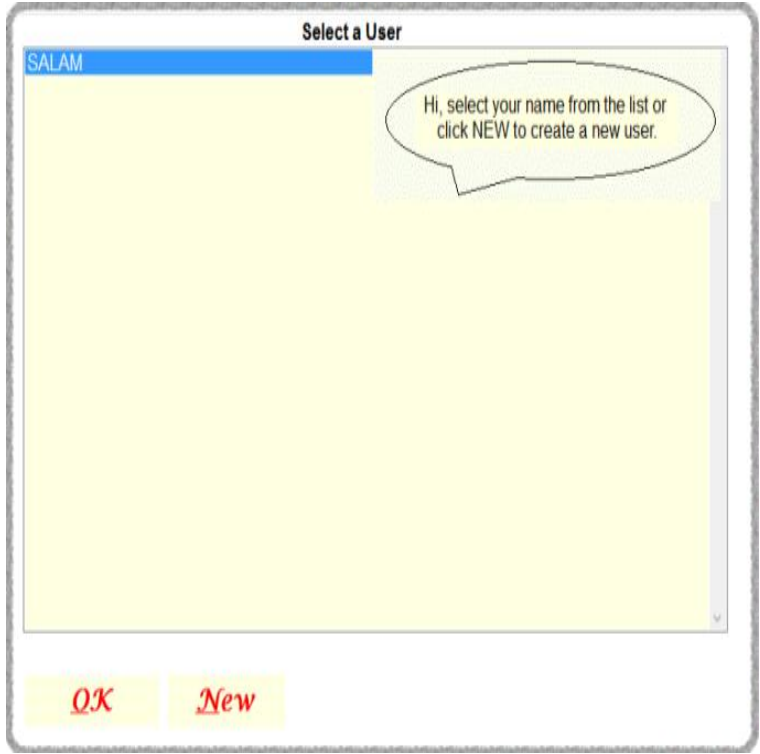

Figure 17: Select a User window

\subsection{Home Window}

When a user logs in, he/she is presented with a list of actions/activities on the Home window to select/undertake. Figure 18 shows the Home window.

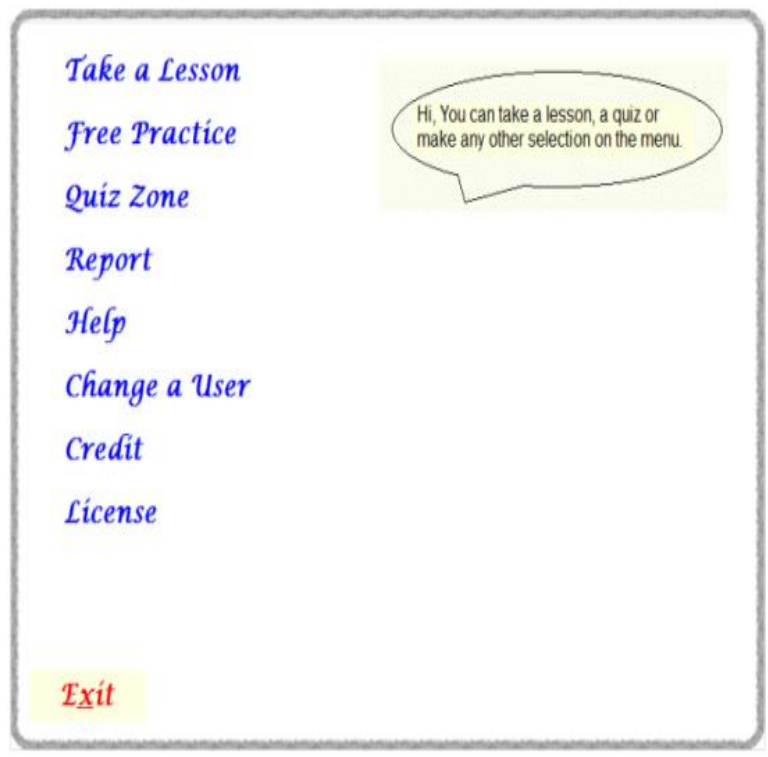

Figure 18: Home window

\subsection{The Lesson Window}

The lesson window contains a series of lessons and exercises designed get the user conversant with the type of abacus select and to teach the user how to perform setting, addition, subtraction, multiplication and division operations in the abacus selected. The Lesson window is shown in Figure 19.

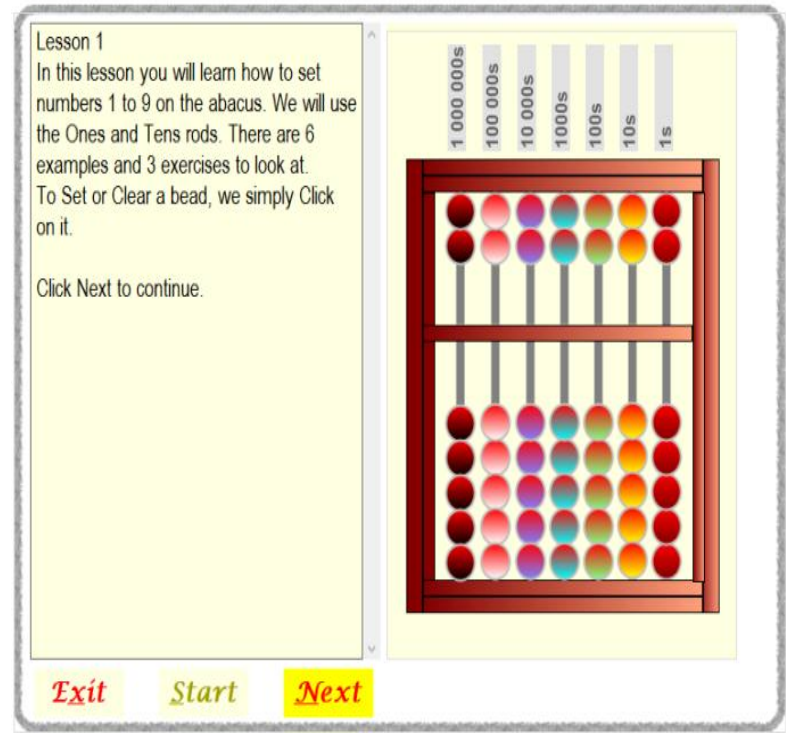

Figure 19: Lesson window

\subsection{The Practice Window}

This window gives the user free practice exercises which are designed to evaluate the users' knowledge of the use of the abacus to perform the various operations of setting, addition, subtraction, multiplication and division of number. This facility also offers the users a "show me" feature that takes the user through a step-by-step procedure to solve problems after wrongly answering a question for five consecutively times.

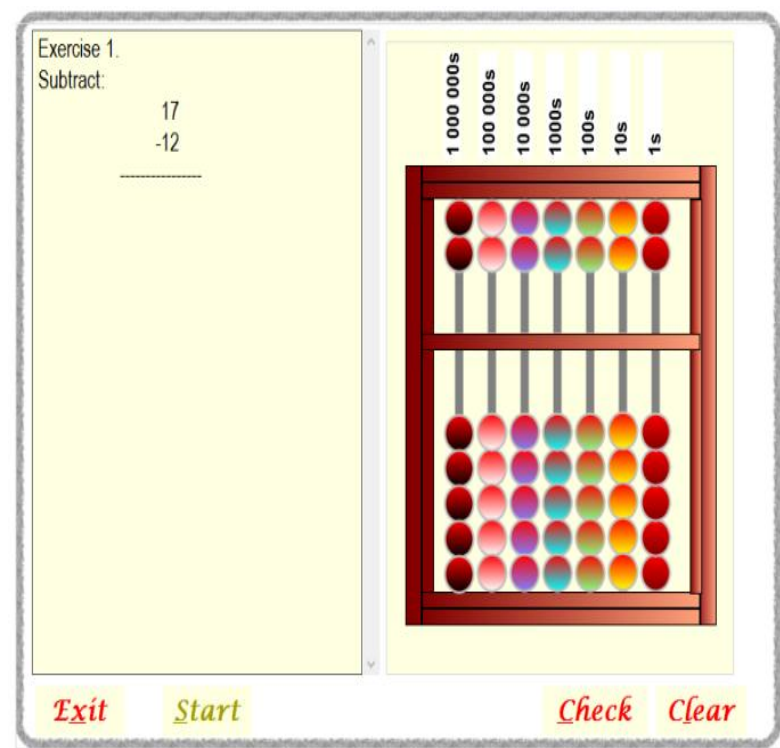

Figure 20: Practice window

\subsection{The Quiz Window}

The Quiz window gives users the opportunity to answer variety of questions in settings, addition, subtraction, multiplication and division in the form of games. 


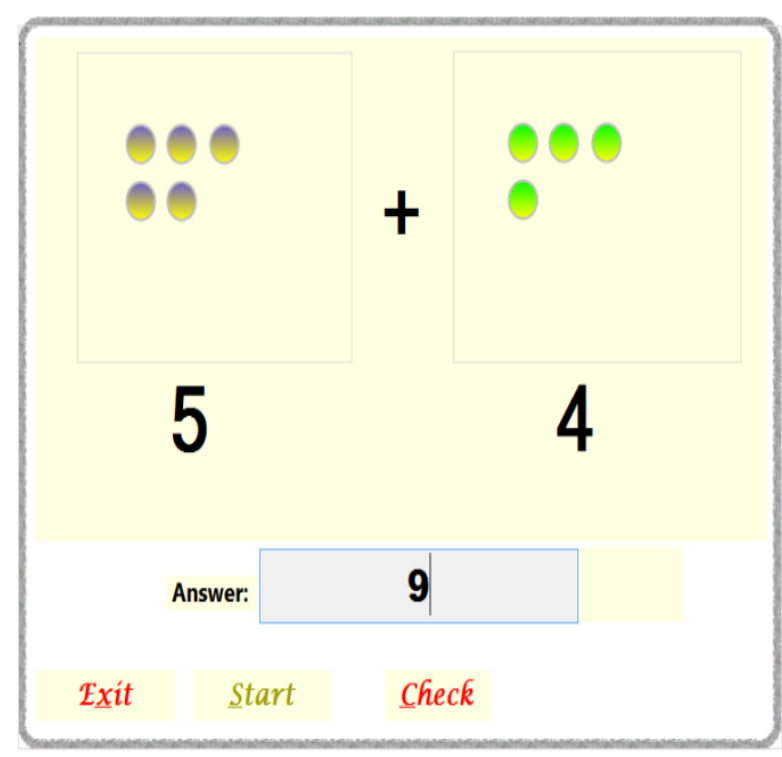

Figure 21: Quiz window

\subsection{The Report Window}

This window gives summary of user activities. It gives information about the current lesson, current practice and rate of answering questions for the user.

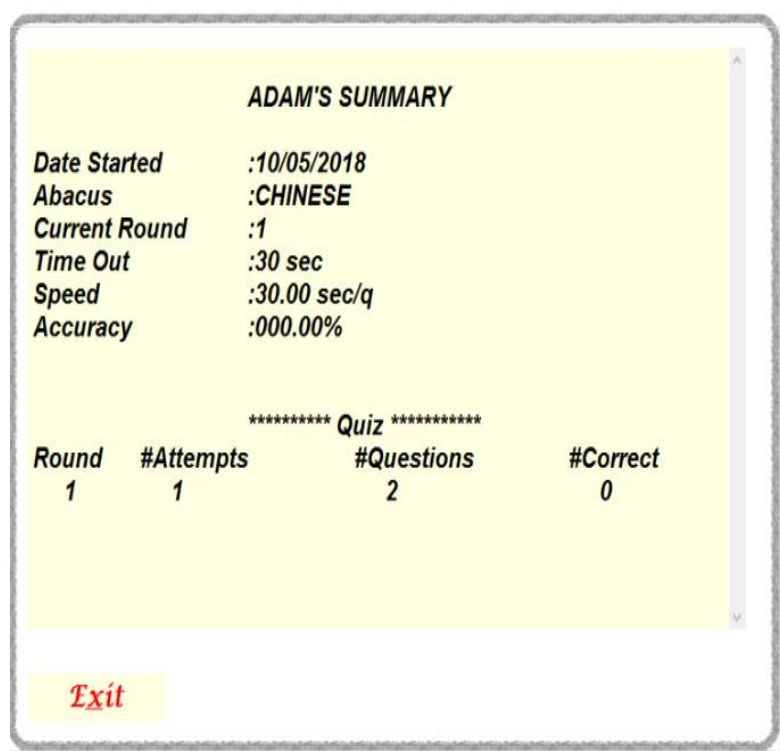

Figure 22: Report window

\subsection{System Use Case Diagram}

A use case diagram is a graphic depiction of the interactions among the elements of a system. It summarizes some of the relationships between use cases, actors, and systems. The use case diagram of the abacus system is shown in Figure 23. In the diagram, the system's boundary (the rectangle) contains the use cases (the ovals). The actor (user of the system) is outside the system's boundary. The relationship between the actor and the use cases are shown using the simple lines drawn between them.

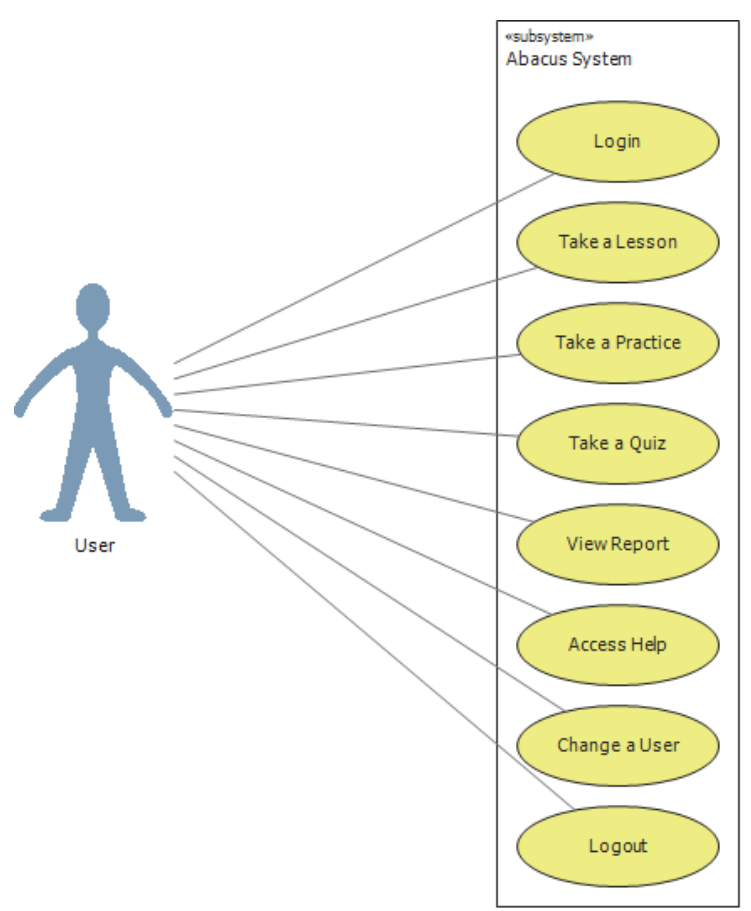

Figure 23: Abacus System Use Case Diagram

\section{IMPLEMENTATION}

Forty-five (45) students were randomly select from classes three, four, and six for assessment of the proposed system. These students were pre-examined (i.e. before the introduction of the abacus system) and post-examination (i.e. after the introduction of the abacus system) in basic mathematics operations of addition, subtraction, multiplication and division. Table 1 show the distribution of the scores obtained by students for the pre-examination.

Table 1. Performance of students before the introduction of Abacus System

\begin{tabular}{|l|l|}
\hline Mark (\%) & Number of students \\
\hline $1-10$ & 0 \\
\hline $11-20$ & 7 \\
\hline $21-30$ & 6 \\
\hline $31-40$ & 8 \\
\hline $41-50$ & 9 \\
\hline $51-60$ & 6 \\
\hline $61-70$ & 7 \\
\hline $71-80$ & 2 \\
\hline $81-90$ & 0 \\
\hline $91-100$ & 0 \\
\hline
\end{tabular}

The results revealed the average performance of the students as approximately $42.38 \%$. This is clearly shown in Figure 24 . 


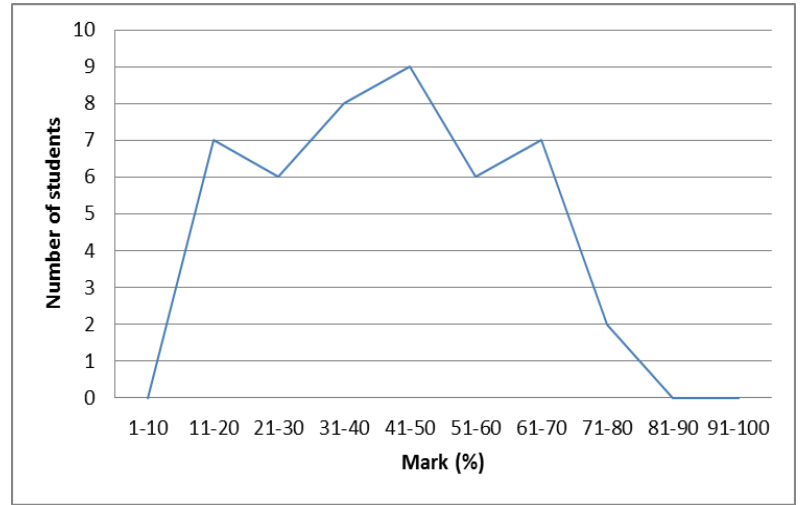

Figure 24: Performance of students before the introduction of Abacus System

The students were introduced to abacus through the teaching of basic mathematics (addition, subtraction, multiplication and division operations). Evaluations of the concepts learnt were done through the use of the Abacus System. Students were taught how to use the system and given ample time to practice on their own. Subsequently, a post-implementation examination was conducted to evaluate student performance. Table 2 summaries the performance of the students in the examination.

Table 2. Performance of students after introductory to Abacus System

\begin{tabular}{|l|l|}
\hline Mark (\%) & Number of students \\
\hline $1-10$ & 0 \\
\hline $11-20$ & 1 \\
\hline $21-30$ & 4 \\
\hline $31-40$ & 4 \\
\hline $41-50$ & 5 \\
\hline $51-60$ & 7 \\
\hline $61-70$ & 11 \\
\hline $71-80$ & 6 \\
\hline $81-90$ & 4 \\
\hline $91-100$ & 3 \\
\hline
\end{tabular}

Figure 25 shows the plot of the performance of students presented in Table 2.

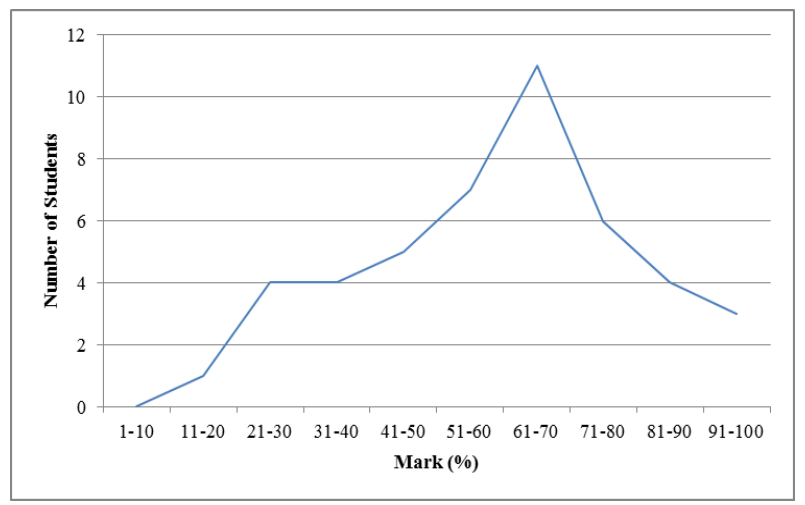

Figure 24: Performance of students after introduction of Abacus System
These results showed an upward improvement of students' performance in basic mathematically to an average of $58.49 \%$. This performance indicates an upward improvement of about 10 percentage points from the pre-introduction examination.

\section{CONCLUSION}

We have developed an Abacus system that helps Ghanaian Basic School students to effectively and efficiently solve arithmetic problems of addition, subtraction, multiplication and division. Child-friendly interfaces and interactive audio facility have been incorporated to facility user understanding of the concept of arithmetic operation through the use of Abacus. Post-implementation results show that students' performance increases by up to 10 percentage points when they are taught to use abacus to perform basic arithmetic operations. It is envisaged that, the proposed system will complement the efforts of Basic School teachers in the teaching of basic mathematics.

Our future work will look at adding more rods to the current system to cater for numbers up to a trillion. We will also look at incorporating finger movement techniques as substitute to moving beads on the abacus to add faster arithmetic operations.

\section{REFERENCES}

[1] David, B. 2004. Soroban Abacus Handbook. Rev 1.03 June 8, 2004

[2] David, B. 2005. Soroban Abacus Handbook. Rev 1.05 March 2, 2005

[3] Totton, H. and Gary, F. The Bead Unbaffled. Accessed on April 10, 2018 from: http://webhome.idirect.com/ totton/abacus/pages.htm\#R evision 1

[4] The Abacus: A Brief History. Accessed on May 1, 2018 from: https://www.ee.ryerson.ca/ elf/abacus/history.html

[5] A Brief Introduction to the Abacus. Accessed on April 20, 2018 from: https://www.ee.ryerson.ca/ elf/abacus/intro.html

[6] Kojima, T. 1954. The Japanese Abacus: Its Use and Theory Tokyo: Charles E. Tuttle, 1954.

[7] Kojima, T. 1963. Advanced Abacus: Japanese Theory and Practice Tokyo: Charles E. Tuttle, 1963.

[8] The Japanese Chamber of Commerce \& Industry. Soroban, the Japanese abacus it's use and practice. Tokyo: Charles E. Tuttle, 1967.

[9] Tani, Y. 1964. The Magic Calculator, the way of the abacus. Japan Publications Trading Co, 1964

[10] Lutjens J. The Abacus - One of the oldest Calculation Devices. Accessed on April 20, 2018 from: https://mafiadoc.com/the-abacus-one-of-the-oldestcalculation-devices-_59dd12541723dda847f526a2.html

[11] "Java" Chinese Abacus. Accessed on May 5, 2018 from http://www.mandarintools.com/abacus.html 